\title{
Impaired X-CGD T cell compartment is gp91phox-NADPH oxidase independent
}

\author{
Maria Chiriaco $^{\text {a,b, } *}$, Fabio Casciano ${ }^{\text {a,b,c }}$, Gigliola Di Matteo ${ }^{\text {a,b }}$, Berhard Gentner ${ }^{\text {d }}$, Alessia Claps ${ }^{\text {a,b }}$, \\ Silvia Di Cesare ${ }^{\mathrm{a}, \mathrm{b}}$, Nicola Cotugno ${ }^{\mathrm{a}, \mathrm{b}}$, Patrizia D'Argenio ${ }^{\mathrm{a}, \mathrm{b}}$, Paolo Rossi ${ }^{\mathrm{a}, \mathrm{b}}$, \\ Alessandro Aiuti ${ }^{\mathrm{e}}$, Andrea Finocchi ${ }^{\mathrm{a}, \mathrm{b}, *}$ \\ a Department of Systems Medicine, University of Rome Tor Vergata, Rome, Italy \\ ${ }^{\mathrm{b}}$ Unit of Immunology and Infectious Diseases, Bambino Gesù Children Hospital, Rome, Italy \\ c Department of Morphology, Surgery and Experimental Medicine and LTTA Centre, University of Ferrara, Ferrara, Italy \\ d San Raffaele Telethon Institute for Gene Therapy (SR-TIGET)/Hematology and Bone Marrow Transplantation Unit, IRCSS Ospedale San Raffaele, Italy \\ e San Raffaele Telethon Institute for Gene Therapy (SR-TIGET)/Vita-Salute San Raffaele University/Pediatric Immunohematology and Bone Marrow Transplantation Unit, \\ IRCSS Ospedale San Raffaele, Milan, Italy
}

\section{A R T I C L E I N F O}

\section{Article history:}

Received 13 April 2017

Received in revised form 19 December 2017

accepted with revision 29 January 2018

Available online 3 February 2018

\section{Keywords:}

CGD

T lymphocytes

TCRV-beta repertoire

gp91phox

NADPH oxidase

\begin{abstract}
A B S T R A C T
Chronic granulomatous disease (CGD) is a phagocytic disorder characterized by a defective production of reactive oxygen species (ROSs). Although infections and granuloma formation are the most common manifestations in CGD patients, a significant number of patients experienced autoimmunity and inflammatory diseases suggesting that adaptive immune abnormalities might be involved.

Here we investigated T-cell compartment and showed that CGD patients had a skewed TCRV-beta distribution in CD8 + T cells, particularly in older patients, and a reduced proliferative responses toward mitogens compared to healthy donors (HD). Afterwards we studied the role of gp91phox protein in causing these alterations and demonstrated that human T cells do not express gp91phox and TCR-stimulated ROS generation is gp91phox-NADPH oxidase independent. Finally, we proved that the NADPH oxidase is not active in the T cell compartment even when forcing gp91phox expression transducing T cells from X-CGD and HD with a SIN lentiviral vector (LVV) encoding the gp91phox cDNA.
\end{abstract}

(c) 2018 Elsevier Inc. All rights reserved.

\section{Introduction}

Chronic granulomatous disease (CGD) is a rare phagocytic disorder caused by defects in any one of genes encoding the NADPH oxidase subunits. CGD is genetically heterogeneous and is characterized by an autosomal recessive (AR-CGD; p22phox, p47phox, p67phox) or $\mathrm{X}$-linked (X-CGD; gp91phox) inheritance. The X-linked CGD is the most frequent form ( $65 \%$ of cases described) and clinically it is more severe than AR-CGD [1].

The oxidase complex plays a key role in both antimicrobial host defence and inflammation, through the production of reactive oxidative species (ROS) essential for the killing of bacteria, fungi and parasites [1]. Although granulocytes in CGD patients are able to engulf the

Abbreviations: CGD, Chronic Granulomatous Disease; NADPH, Nicotinammide adenina dinucleotide fosfato; TCR, T-cell reception; ROS, Reactive oxygen species; PBMC Peripheral blood mononuclear cells; SIN-LVV, Self-inactivating lentiviral vector.

* Corresponding authors at: Department of Systems Medicine, University of Rome Tor Vergata, Rome, Italy/Unit of Immunology and Infectious Diseases, Bambino Gesù Children Hospital, Rome, Italy.

E-mail addresses: maria.chiriaco@opbg.net (M. Chiriaco), andrea.finocchi@uniroma2.it (A. Finocchi). pathogens, failure to produce ROS prevents the clearance of the microorganisms. As a consequence, CGD patients are susceptible to recurrent life-threatening pyogenic infections, particularly those caused by catalase-positive bacteria and fungi. Moreover, CGD often have poor wound healing and chronic inflammation leading to granuloma formation [2]. Given the improved treatment of infections and survival in CGD patients, nowadays the increased susceptibility to inflammatory and autoimmune diseases has become an increasing relevant issue in patients. At the moment, Systemic Lupus- and Discoid Lupus-Erythematosus, thrombocytopenic purpora, arthritis and inflammatory bowel disease have been often described in CGD patients, suggesting a protective role of ROS in autoimmune disease [3-5]. The evidence that, in addition to phagocytes, other cells of the innate and adaptive immune system such as B lymphocytes and dendritic cells (DC) are able to produce moderate levels of oxygen radicals, highlights the key role that ROS have in regulating immune system interaction and function [6,7]. Particularly we showed in CGD patients that the lower frequency of memory B-cells and higher frequency of naïve B-cells, with their reduced ability to proliferate and differentiate upon in vitro BCRstimulation, are dependent on the defect in BCR-derived ROS production by NADPH oxidase complex [8]. On the other hand, the presence of functional NADPH oxidase complex in T lymphocytes remains 
controversial. Although T cells contain low levels of ROS that occur upon T-cell receptor (TCR) stimulation, it is unclear if oxygen species are actually derived from respiratory burst enzymes or from mitochondria and how they affect $\mathrm{T}$ cell functionality [9-11]. Interestingly, T cell lymphopenia and reduced proliferation to mitogens in CGD patients [12], suggest a link between ROS production and the susceptibility to infections. However, how a molecular defect in CGD could influence T-cell function and consequently play a role in the pathogenesis of the disease remains poorly understood.

Here, we performed a phenotypic and functional characterization of Tcell compartment in CGD patients and investigated the role of gp91phoxNADPH oxidase in the production of ROS in T cells. We analysed lymphocyte proliferation upon TCR-stimulation and TCR-V $\beta$ repertoire and found a reduced proliferative capacity in X-CGD together with age-related skewed distribution of TCR repertoire in CD8 + T cells. Moreover, we demonstrated that after TCR stimulation ROS were produced regardless of the gp91phox-NADPH oxidase expression. Furthermore, transducing with a SIN lentiviral vector (LVV) encoding the gp91phox protein [13] T cells collected from X-CGD and HD subjects, we proved that forced gp91phox expression does not induce NADPH oxidase activity.

\section{Material and methods}

\subsection{Patients and informed consent}

The study enrolled nine male CGD patients ( 10 gp91phox-CGD and 1 p22phox-CGD) during follow up procedures. All patients analysed did not express the protein $\left(\mathrm{gp} 91^{\circ}\right.$ or $\left.\mathrm{p} 22^{\circ}\right)$. Blood samples were taken from HD and CGD after obtaining informed consent following standard ethical procedures with approval of the Children's Hospital Bambino Gesù Ethical Committee. Both HD and CGD were free of infection at follow-up and patients were off corticosteroid and immunosuppressive therapy at the time of the blood sampling or previously. Clinical and molecular data of CGD patients are summarized in Table 1.

\subsection{T-cell proliferation assay}

Peripheral blood mononuclear cells (PBMCs) from 11 CGD patients and 8 age-related HDs were obtained by centrifugation using FicollPaque (Euroclone) and stimulated with PHA $5 \mu \mathrm{g} / \mathrm{ml}$ (Sigma Chemical, Milan, Italy) or OKT3 $3 \mu \mathrm{l} / \mathrm{ml}$ (stock supernatant obtained from OKT3 cell line, ATCC-Sigma, MI, Italy) for $72 \mathrm{~h}$. PBMC from the same sample were cultured for 3 and 6 days without any stimulus as negative control. $\mathrm{T}$ cells proliferation was investigated as described [14] and results were indicated as stimulation index (SI), defined as the ratio between the average counts of stimulated and unstimulated PBMC. Lymphoproliferation towards mitogens, was considered positive if PHA $>35.000 \mathrm{cpm}$ or OKT3 $>25.000 \mathrm{cpm}$.

\subsection{TCR spectratyping}

PBMC from 11 patients and 8 age related HD, were fractionated into $\mathrm{CD}^{+}$and $\mathrm{CD}^{+}$subsets using anti-CD4+ or anti-CD8+ monoclonal antibody-coupled magnetic beads (Dynal AS, OSLO, Norway) following manufacturer's instructions. The purity of the two subsets was $>95 \%$. Total RNA was extracted by TRIzol ${ }^{\circledR}$ (Gibco-BRL/Life Technologies) in accordance with the manufacturer's instructions. T cell receptor CD4 and CD8 V beta repertoire were investigated essentially as described [15]. Particularly, we defined collected data as polyclonal (p), polyclonal altered (pa) and skewed/perturbed (sk) patterns, respectively with a Gaussian "bell shaped distribution", a non-Gaussian-like distribution and with a skewed profiles consisted of 1-4 peaks or a multipeak pattern with one solitary peak $>50 \%$ of the total area or one or more deleted peaks.

\subsection{Flow-cytometry studies}

Cells were stained with antibodies against: anti-human CD3-PERCP (BD), anti-human gp91 ${ }^{\text {phox }}$-FITC (anti-flavocytochrome B558, clone 7D5) (MBL, Medical and Biological Laboratories Co., Japan), CD45RAAPCH7 (BD), CD4-APC (BD), CD8-APC (BD). Intracellular staining was performed using cytofix-cytoperm (BD) in accordance with the manufacturer's instructions. FACS Canto II (Becton-Dickinson, USA) and Flowjo (Tree Star, Inc.) were used to collect and analyze the data obtained.

\subsection{Detection of ROS in $T$ cells}

T cells were isolated from PBMC using PanT Cell Isolation Kit (Miltenyi Biotec) and stimulated with OKT3 $(10 \mu \mathrm{l} / \mathrm{ml}), \alpha C D 28(10 \mu \mathrm{g} / \mathrm{ml})(\mathrm{BD})$ or phorbol 12-myristate 13-acetate (PMA) (Sigma) for $48 \mathrm{~h}$. To investigate ROS production cells were further incubated for $10 \mathrm{~min}$ at $37^{\circ} \mathrm{C}$ in water bath in the dark with $0.5 \mu \mathrm{M} 2^{\prime}, 7^{\prime}$-dichlorodihydrofluorescein diacetate (DCFDA; Invitrogen) or with dihydrorhodamine (DHR) 123 DHR (Diidrorhodamine). Oxidative reactions were stopped and fixed with paraformaldehyde in phosphate buffer saline. ROS emission was measured at 530/30 using $488 \mathrm{~nm}$ laser excitation on a FACSCanto (BD) with DIVA software. Data were subsequently analyzed with the FlowJo software (Tree Star, Inc.) and reported as Stimulation Index (SI) calculated as ratio between mean of fluorescent intensity (MFI) of stimulated cells and MFI of un-stimulated cells.

\subsection{Transduction}

PBMCs from X-CGD patients and HD were cultured in presence of OKT3 $(10 \mu \mathrm{l} / \mathrm{ml}) / \alpha C D 28(10 \mu \mathrm{g} / \mathrm{ml}$;BD)/IL2 (100 UI/ml, SIGMA) for 2 days and then washed, and transduced with LV PGK.gp91 at MOI 50 in presence of IL2 (100 UI/ml) and Polybrene ( $4 \mu \mathrm{g} / \mathrm{ml})$ (SIGMA). PGK.

Table 1

Clinical and molecular data of CGD patients enrolled for the study.

\begin{tabular}{|c|c|c|c|c|c|c|c|}
\hline Patients & Gender & $\begin{array}{l}\text { Age } \\
(\mathrm{y})\end{array}$ & DNA change & $\begin{array}{l}\text { Protein } \\
\text { expression }\end{array}$ & NADPH function & Severe infections & $\begin{array}{l}\text { Noninfectious } \\
\text { complications }\end{array}$ \\
\hline Pt1 & Male & 18 & del Xp11.4 & $\mathrm{Gp} 91^{\circ}$ & No residual activity & Lung abscess, lymphadenitis, pyodermitis (Serratia) & IBD \\
\hline Pt2 & Male & 11 & c. $1357 \mathrm{~T}>\mathrm{A}$ & Gp91 & No residual activity & & IBD \\
\hline Pt3 & Male & 30 & c. $1006 \mathrm{G}>\mathrm{T}$ & $\mathrm{Gp} 91^{\circ}$ & No residual activity & Sepsis (Staphylococcus), Osteomyelitis (C. albicans) & IBD \\
\hline Pt4 & Male & 3 & c. $83 \mathrm{G}>\mathrm{A}$ & $\mathrm{Gp} 91^{\circ}$ & No residual activity & Liver abscess, meningitis (Mycobacterium tuberculosis) & \\
\hline Pt5 & Male & 13 & c. $1076 G>C$ & $\mathrm{Gp} 91^{\circ}$ & No residual activity & Lung aspergillosis, suppurative lymphadenitis & \\
\hline Pt6 & Male & 10 & del Xp11.4 & $\mathrm{Gp} 91^{\circ}$ & No residual activity & Lung aspergillosis, lung abscess & $\mathrm{CE}$ \\
\hline $\mathrm{Pt}^{*}$ & Male & 2 & c. $252 \mathrm{G}>\mathrm{A}$ & Gp91 ${ }^{\circ}$ & No residual activity & & \\
\hline Pt8 & Male & 5 & c.1287delT/c.1290delC & $\mathrm{Gp} 91^{\circ}$ & No residual activity & Lung aspergillosis & $\begin{array}{l}\text { Granulomatous } \\
\text { Cistitis }\end{array}$ \\
\hline Pt9 & Male & 6 & c.295-301del GTGCCCG & $\mathrm{p} 22^{\circ}$ & No residual activity & BCG infection, liver abscess, lymphadenitis & \\
\hline Pt10 & Male & 12 & c.338-2A > C/p.Cys85ArgFsX15 & $\mathrm{Gp} 91^{\circ}$ & No residual activity & Osteomyelitis, pyodermitis, lung pneumoniae & IBD \\
\hline Pt11 & Male & 42 & c. $252 \mathrm{G}>\mathrm{A}$ & $\mathrm{Gp} 91^{\circ}$ & No residual activity & Lung pneumoniae, liver abscess, pyodermitis & \\
\hline
\end{tabular}

IBD (inflammatory bowel disease); $\mathrm{CE}$ (celiac disease).

* Pt7 is the nephew of Pt11. 
Table 2

Phenotypic investigations of CD4 + and CD8 + T cell subsets.

\begin{tabular}{|c|c|c|c|c|c|}
\hline \multirow[t]{2}{*}{ Patients } & \multirow[t]{2}{*}{$\operatorname{Age}(y)$} & \multicolumn{4}{|c|}{$\begin{array}{l}\text { T cells memory/naive phenotype }{ }^{* *} \text { (gated on CD } 45+\text { CD3 + } \\
\text { cells) }\end{array}$} \\
\hline & & $\% \mathrm{CD} 4 / \mathrm{RA}+$ & $\% \mathrm{CD} 4 / \mathrm{RO}+$ & $\% \mathrm{CD} 8 / \mathrm{RA}+$ & $\% \mathrm{CD} 8 / \mathrm{RO}+$ \\
\hline Pt1 & 18 & 37 & 44 & 73 & 15 \\
\hline Pt2 & 11 & 70 & 30 & 78 & 22 \\
\hline Pt3 & 30 & 35 & 65 & 49 & 51 \\
\hline Pt4 & 3 & nd & nd & nd & nd \\
\hline Pt5 & 13 & nd & nd & nd & nd \\
\hline Pt6 & 10 & 64 & 36 & 55 & 44 \\
\hline Pt7 & 1 & 88 & 8 & 92 & 8 \\
\hline Pt8 & 5 & 61 & 39 & 60 & 40 \\
\hline Pt9 & 6 & 43 & 57 & 40 & 60 \\
\hline Pt10 & 12 & 59 & 41 & 56 & 44 \\
\hline Pt11 & 42 & 27 & 73 & 49 & 51 \\
\hline \multirow[t]{4}{*}{ HD } & \multicolumn{5}{|c|}{ **Normal range for age from Shearer WT et al., JACI 2003} \\
\hline & $2-6 y$ & 71 & 16 & 86 & 9 \\
\hline & $6-12 y$ & 59 & 21 & 80 & 12 \\
\hline & $>12 y$ & 53 & 28 & 79 & 13 \\
\hline
\end{tabular}

gp91 LVV designed, production and efficiency of transduction on human myeloid and CD34+ cells were previously described [13]. After 7 days of culture, cells were investigated for the presence of gp91phox protein and ROS-generation capacity using DHR and DCFDA substrates. Cells were cultured for 14 days after transduction in order to eliminate non-integrated vector forms and vector copy number per genome (VCN) were quantified by quantitative Real-Time PCR (Q-PCR) as described [13].

\subsection{Statistical analysis}

Collected data were processed using Prism 5 (GraphPad Software, San Diego, CA). The mean values of repeated immunological parameters were calculated. The Shapiro-Wilcoxon test was used to evaluate the Gaussian distribution of overall immunological parameters. Statistical comparisons between patients and HD were calculated with nonparametric analyses (one-way ANOVA) when no Gaussian distribution was found and exact $p$ values were obtained, otherwise $t$-students' test and column statistics was used. A significant threshold of $p<0.05$ was used to assess the statistical differences in patients versus healthy control values.

\section{Results}

\subsection{Lymphocytes from CGD patients have reduced proliferative capacity}

Immunological investigations revealed that all CGD patients showed a normal total B- and T-lymphocytes number, but reduced peripheral memory B-cell compartment. An imbalance toward memory phenotype, investigated by the differential expression of CD45 isoforms, for both CD4+ and CD8+ T-cell was observed (Table 2).

In order to investigate the proliferation capacity of PBMCs after mitogen stimulation, cells isolated from CGD patients and HDs were stimulated with PHA or OKT3. The patient's lymphocytes showed a statistically significant reduction of mitogen responsiveness to PHA (SI HD $82.41 \pm 10.4$ vs SI CGD $43.54 \pm 9.3 p=0.027$ ) and OKT3 (SI HD $93.86 \pm 17.2$ vs SI CGD $30.08 \pm 7.19, p=0.027$ ) respect to age-matched healthy donors (Fig. 1).

\subsection{Skewed distribution of CD8-TCR repertoire in CGD patients seems to be influenced by age}

Molecular analysis of T-cell receptor (TCR) repertoire of CGD patients and HDs was studied by measuring the CDR3 heterogeneity length of variable beta regions by spectratyping. Sorted CD4 and CD8 T cells were investigated for 24 TCR-V beta different families.

Results showed that CGD patients presented a normal polyclonal profile in the CD4+ T cells, but a skewed distribution in CD8 + T cells with respect to HD $(p<0.001)$ (Fig. 2B). In order to check if abnormalities in CD8 + T cell populations were age-dependent, the results were reanalysed considering two different sub-groups of patients: pediatric $(\mathrm{nPP}=6$; age $<12)$ and non-pediatric $(\mathrm{nNPP}=5$; age $>12)$. The analysis showed that the distribution profile in CD8 + T cells is significantly $(p<0.05)$ skewed in NPP CGD with respect to HD (Fig. 2C), with no evidence for preferential skewing of particular $\mathrm{V}$ beta subfamily.

\subsection{Human $T$ cells do not express gp91phox and TCR-stimulated ROS generation is gp91phox-NADPH oxidase independent}

To determine if the defect in T cell compartment of CGD patients was linked to NADPH oxidase deficiency, T cells collected from X-CGD patients $\left(\mathrm{gp} 91^{\circ}\right)$ and HDs were studied for the presence of gp91phox on cell surface and intracellular compartment, and for the involvement of an NADPH oxidase in TCR-stimulated reactive oxygen species (ROS) generation.

As expected, the protein is expressed on of neutrophils, monocytes and B-cells' cell surface (100\%) from HD, but it was absent in the subsets from X-CGD patients. Interestingly, the expression of gp91phox protein resulted undetectable on the cell surface of $\mathrm{T}$ cells from both patients ( $n=2$ with missense mutation, and $n=2$ with whole gene deletion) and HDs $(n=6)$ (Fig. 3A).

Since previous studies on gp91phox expression in T cells were performed by intracellular staining [16] we analysed gp91phox using this method. The intracellular staining performed on CD3 cells revealed that percentage and mean fluorescence intensity (MFI) of gp91phox was similar between HD (99\%; MFI = 7558) and both X-CGD with whole CYBB gene deletion $(98 \%$; MFI $=4813)$ and with missense
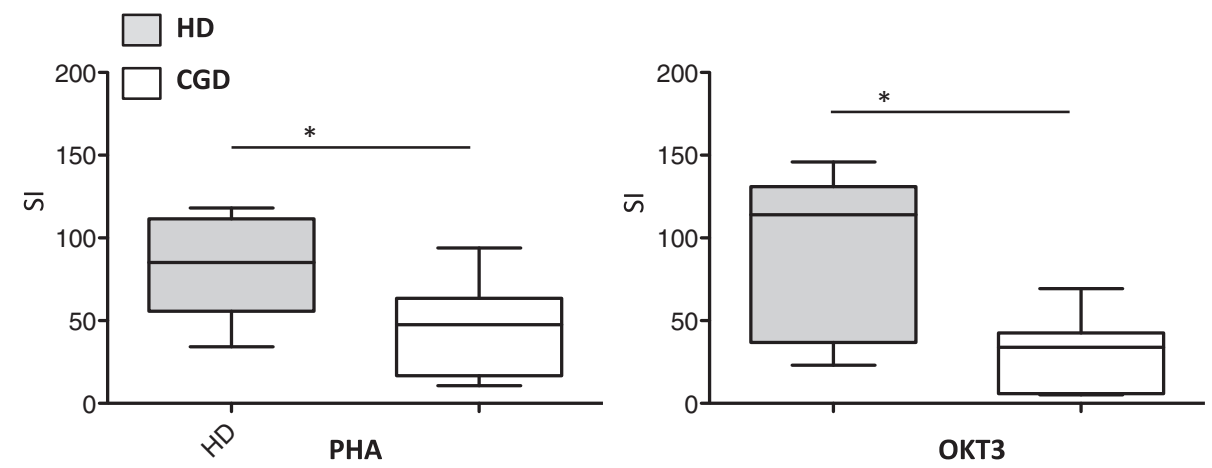

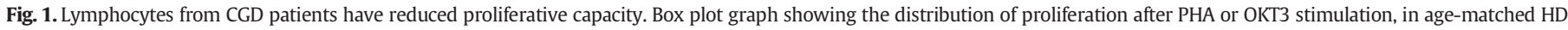

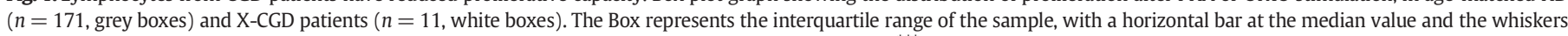
defining the highest and lowest values. Statistical analysis was performed using one-way ANOVA test; ${ }^{* * *} p<0.05$. 
A)

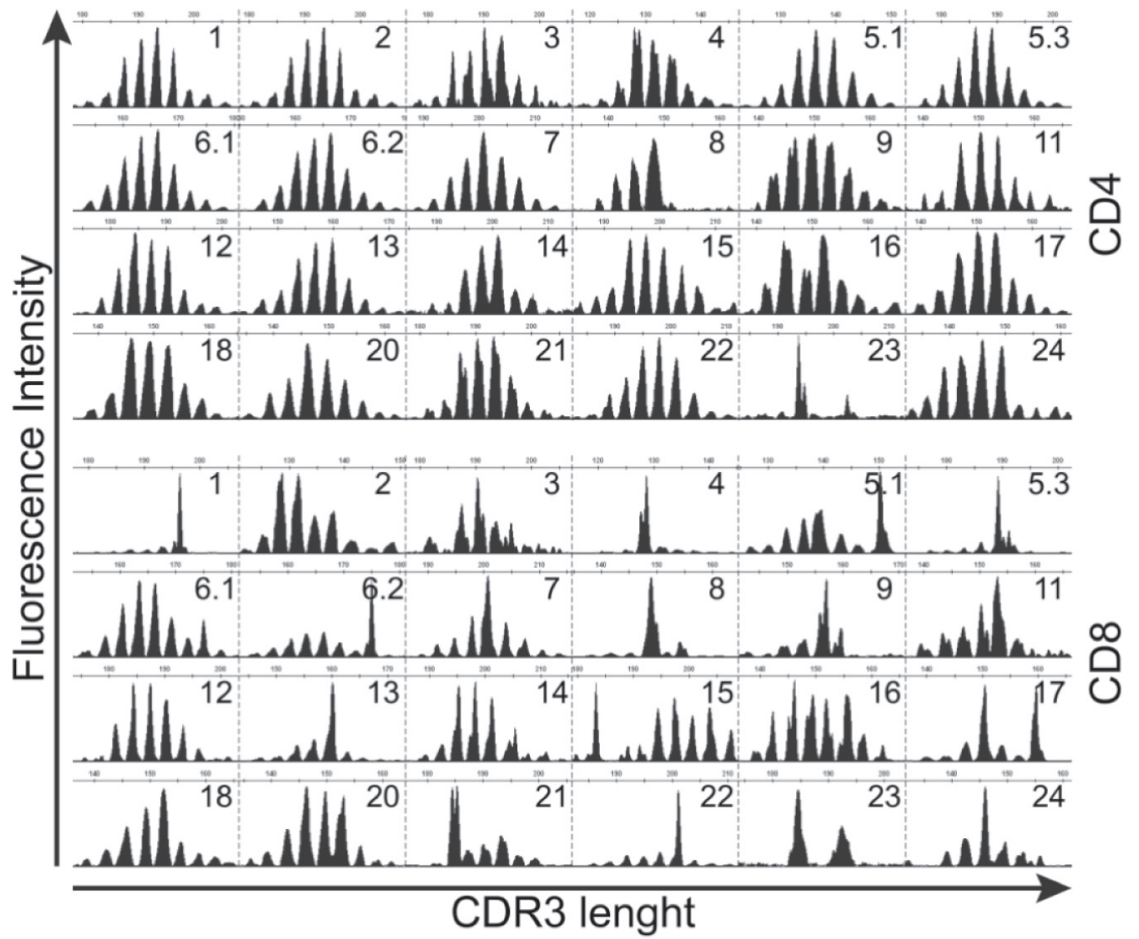

B)

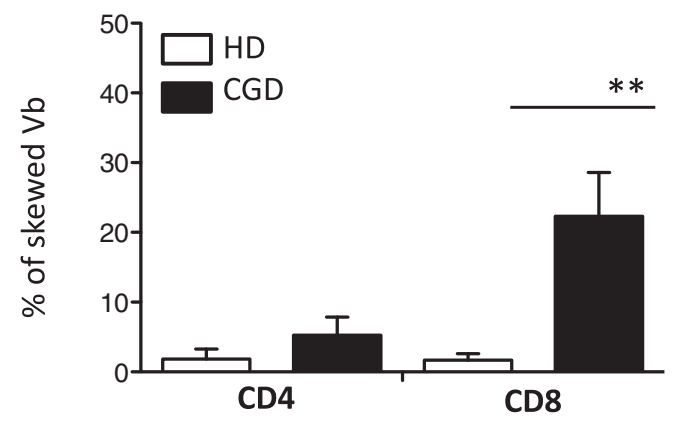

C)

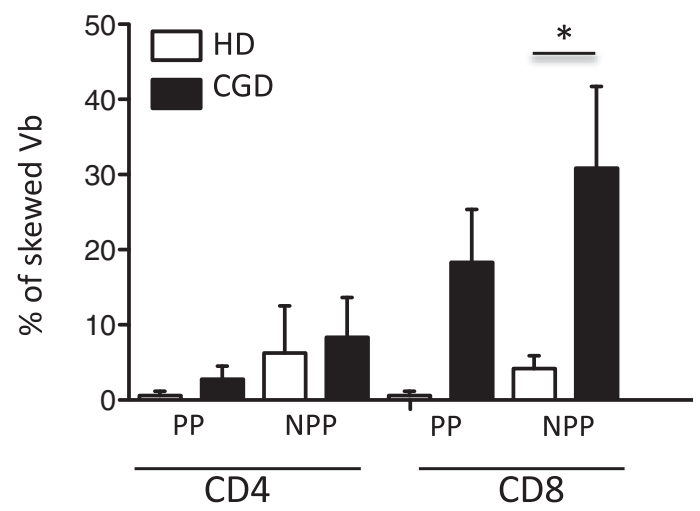

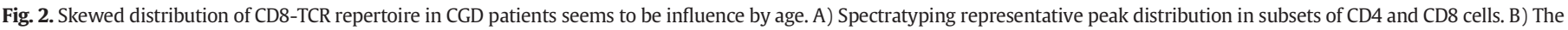

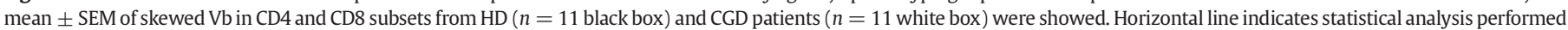

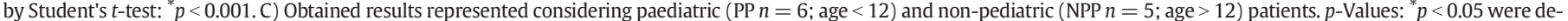
termined by the Student's $t$-test. 
A
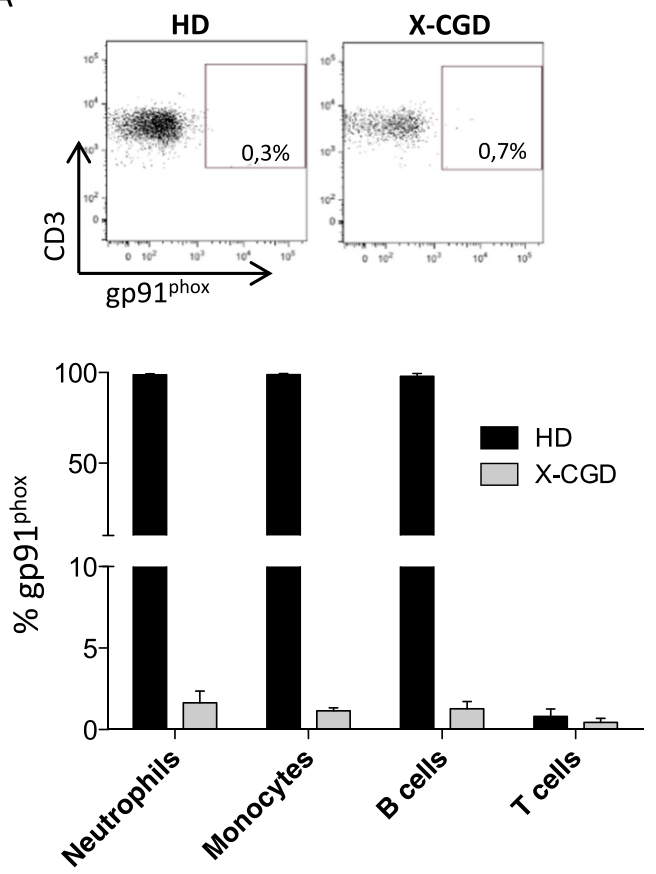

B

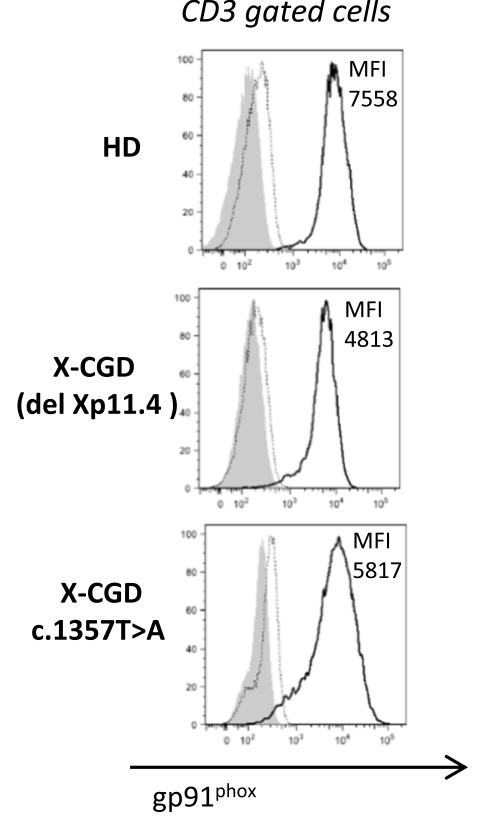

C
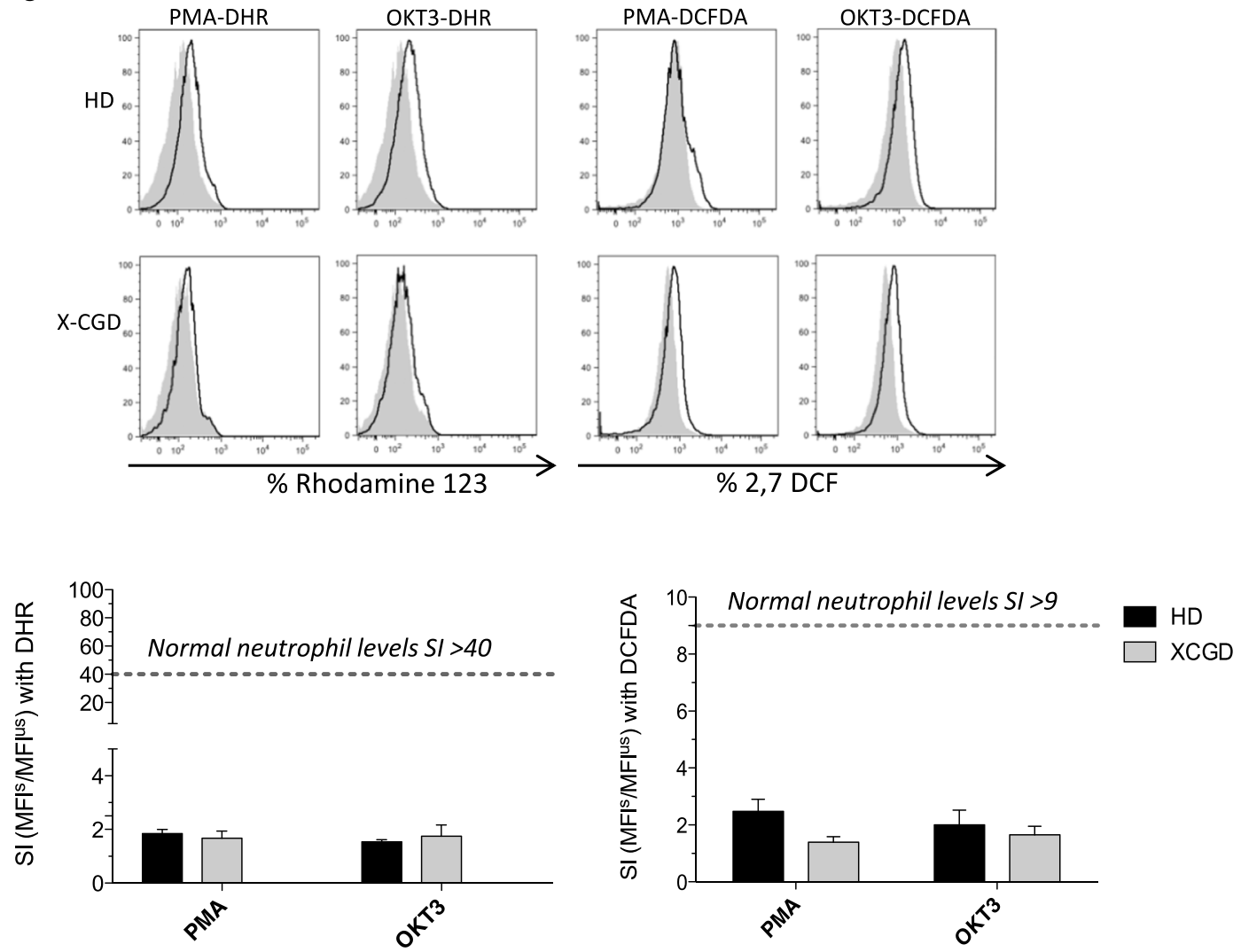

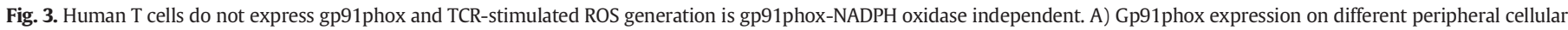

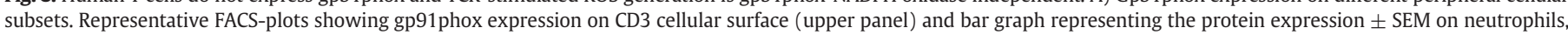

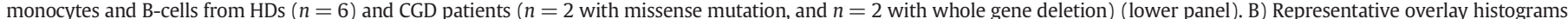

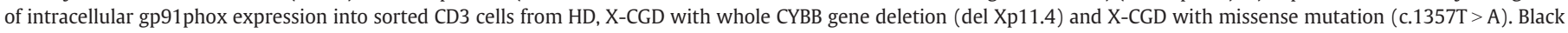

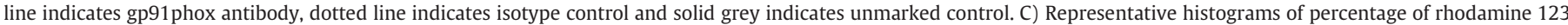

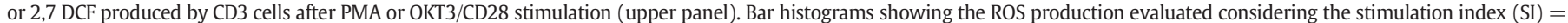
MFI ${ }_{\text {stimulated cells } / M F I}$ unstimulated cells \pm SEM. Normal level evaluated on neutrophils were also showed (lower panels). 
A)

Gated on CD3 cells

A)

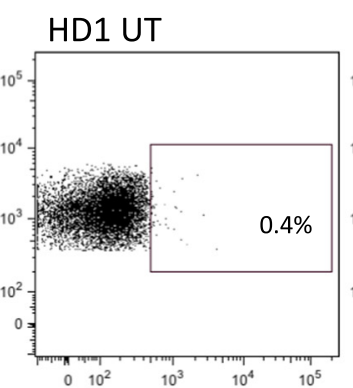

HD1 PGK.gp91

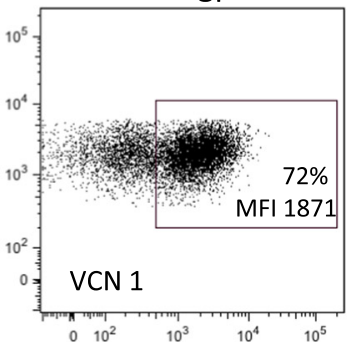

HD2 PGK.gp91
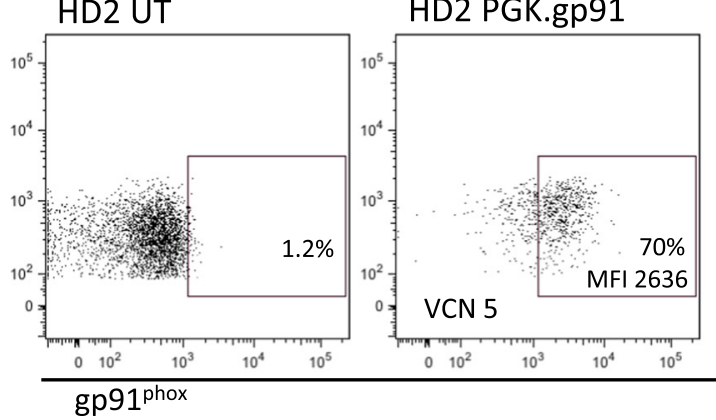

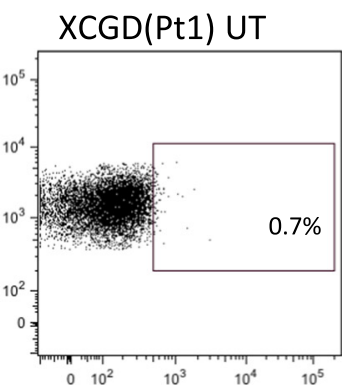

XCGD(Pt1) PGK.gp91

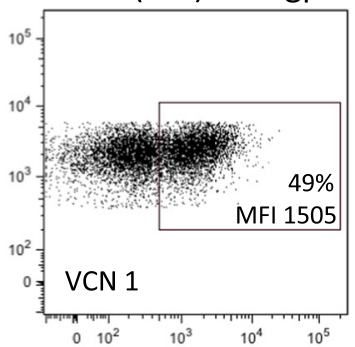

XCGD(Pt2) UT

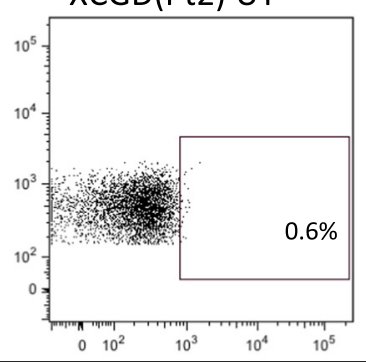

XCGD(Pt2) PGK.gp91

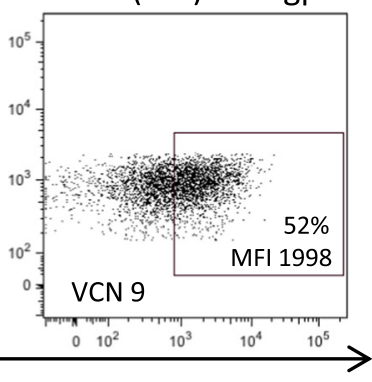

B)

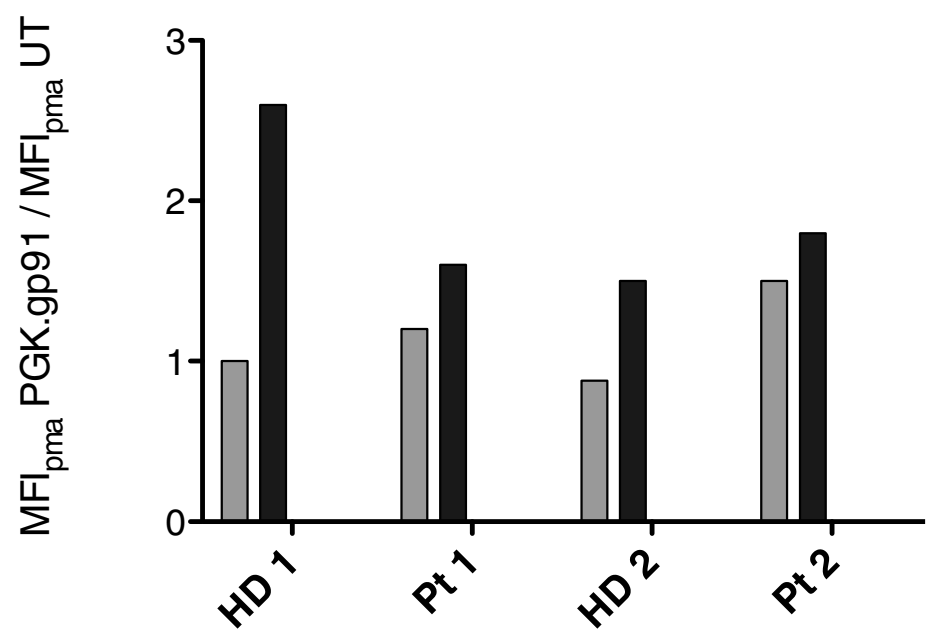

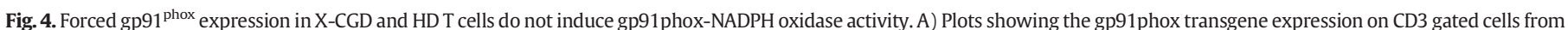

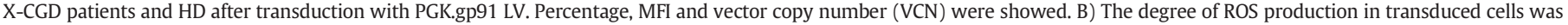
considered as ratio between MFI of PMA stimulated PGK.gp91 transduced cells and MFI of PMA un-transduced cells.

mutation (97\%; MFI = 5817), suggesting that the fluorescence is due to background staining of the gp91phox antibody (7D5) (Fig. 3B).

Next, we investigated the production of oxidants from CD3 cells after nonspecific stimulation phorbol-12-myristate-13-acetate (PMA)mediated and specific OKT3/CD28 TCR-stimulation. We performed the experiments using the dihidrorhodamine 123 (DHR123) and cellpermanent $2^{\prime}, 7^{\prime}$-dichlorodihydrofluorescein diacetate $\left(\mathrm{H}_{2} \mathrm{DCFDA}\right)$ probes, which in presence of ROS are converted respectively to the highly fluorescent rhodamine 123 (directly via $\mathrm{H}_{2} \mathrm{O}_{2}$ ) and $2^{\prime}$, 7' dichlorofluorescein (DCF) (initially via esterase and then via $\mathrm{H}_{2} \mathrm{O}_{2}$ ) [17].
Results showed that both X-CGD and HD cells after prolonged culture with stimuli induced a minimal oxidation of both probes (Fig. $3 \mathrm{C}$ upper panel). In particular, the difference between the stimulation index (SI) evaluated in $\mathrm{HD}(n=7)$ and X-CGD $(n=7)$ is not statistically significant for both probes DHR (SI PMA: $1.8 \pm 0.2$ vs $1.7 \pm 0.5$ and SI OKT3: $1.5 \pm$ 0.2 vs $1.8 \pm 0.8$ ) and DCFDA (SI PMA: $3 \pm 0.7$ vs $1.4 \pm 0.2$ and SI OKT3: $2.5 \pm 0.7$ vs $1.7 \pm 0.3$ ), despite a tendency to a slight increase of the oxidation detected by the first probe in HD rather than in X-CGD (Fig. 3C lower panels). These results suggested that ROS generated after TCRstimulation $(\mathrm{SI}<3)$ were independent from NADPH oxidase activity. 
3.4. Forced gp91phox expression in X-CGD and HD T cells do not induce gp91phox-NADPH oxidase activity

To investigate if the forced gp91phox expression in T cells was able to induce the NADPH oxidase activity, T cells from X-CGD patients and HDs were cultured in presence of OKT3/CD28/IL2 and transduced (Multiplicity of Infection-MOI 50) with a LV expressing human codon optimized gp91phox cDNA under the control of a ubiquitous cellular promoter (PGK.gp91).

After 7 days of culture the gp91phox transgene protein was presented on all transduced cells (Fig. 4A) with variable expression levels in both X-CGD (49-52\%; VCN = 1-9) and HD (72-70\%; VCN = 1-5). We tested the functionality of the oxidase activity and found that by DHR assay both HD and patients did not have the activity of NADPH oxidase $\left(0,88<\mathrm{MFI}_{\text {pma }}\right.$ PGK.gp91/MFI $\left.\mathrm{Mma}_{\mathrm{u}} \mathrm{UT}<1.5\right)$. However by DCFDA assay we found a slight increase of the oxidase activity $\left(1.5<\mathrm{MFI}_{\text {pma }}\right.$ PGK.gp91/MFI pma $_{\text {UT }}<2.6$ ) in both HD and patients probably caused by the influence of cytochrome $c$ levels that could give a signal without any change in cellular peroxide levels. These results suggested that the NADPH oxidase complex is not functional in T cells.

\section{Discussions and conclusion}

Phagocytes, primarily neutrophils, have been extensively studied in the pathogenesis of CGD because of their ability to kill and clear bacteria and fungi during the infection through the production of ROS by the NADPH oxidase complex. In the last years various studies showed that several non-phagocytic leukocytes, such as B-lymphocytes, dendritic and NK cells, are capable of producing moderate amount of ROS, suggesting that ROS are not only harmful and mediators of oxidative stress, but also have immune regulatory functions, especially when produced in lower amounts $[1,18]$.

In this regard we have recently demonstrated that CGD patients have lower memory and higher naïve B-cells counts related to impaired long-term memory in CGD, and that impaired B-cells are dependent on defective ROS-production by BCR [8]. The ability of T lymphocytes to generate ROS upon TCR stimulation has been also investigated by Belikov AV et al. [19] that showed how T lymphocytes exhibit a very small burst production reactive oxygen products in response to mitogen stimulus, and that a portion of this burst is missing in mouse and human CGD T lymphocytes.

In contrast other groups were unable to detect any ROS production by $\mathrm{T}$ lymphocytes following TCR stimulation [12]. It is likely that ROS influence T-lymphocytes signalling, however it is not clear whether T-lymphocytes produce ROS themselves or whether ROS in particular $\mathrm{H}_{2} \mathrm{O}_{2}$ diffuse from APCs to T-lymphocytes [20]. Interestingly, slight abnormalities in T-lymphocytes have been reported in CGD patients, including diminished T-cell number, a tendency to reduced proliferative responses and diminished expression of CD40L on T cells, suggesting a possible role of ROS in T cells [21]. In order to better define the role of $T$ cells in CGD patients we performed a careful phenotypic and functional characterization of T-cell and we evaluated the role of gp91phox-NADPH oxidase in the production of ROS by $\mathrm{T}$ cells.

Our data showed that CGD T lymphocytes have significantly reduced proliferative response toward mitogens. Furthermore, we demonstrated significantly higher skewing of TCR-V beta in pheripheral CD8 + T cells among CGD patients compared to healthy controls, with no evidence for preferential skewing of particular $\mathrm{V}$ beta subfamily. Particularly, the frequency of skewed TCR-V $\beta$ subfamilies in older patients was significantly higher than that observed in younger patients and HD. Because none of the patients had clinical and laboratory evidence of acute infection at the time of sample collection, we conclude that these alterations reflect a stable state of the TCR repertoire, consolidated over time, in CGD patients.
The clinical relevance of a contracted $\mathrm{T}$-cell repertoire and reduced $\mathrm{T}$ cell proliferation in CGD is unclear; $\mathrm{T}$ cell compartment has been investigated in some studies. Although infections and granuloma formation remain the most common manifestations in patients with CGD, there is a significant subset of CGD patients who experienced a broad variety of autoimmune and inflammatory disease suggesting the possibility of concomitant adaptive immune abnormalities [22].

Whether the decreased and skewed TCR VB diversity might be involved in the pathogenesis of immunodeficiency and autoimmunity or might be the consequence of chronic antigen exposure remains to be clarified in patients with CGD as well as underlying molecular mechanisms.

In order to define if impaired TCR-expression and function were dependent on defective gp91phox expression, we investigated the involvement of NADPH-oxidase in the generation of ROS in TCRstimulated T cells from CGD patients and HD. Initially we assessed that human T cells didn't express gp91phox protein on the cellular surface and the production of ROS is gp91phox-NADPH oxidase independent. Finally, forcing the expression of gp91phox protein transducing T cells from X-CGD patients and HD with LV.PGK.gp91, we showed that gp91phox-NADPH oxidase complex did not generate ROS, probably for the absence of other NADPH oxidase subunits [23].

In summary our results indicate that X-CGD patients a skewed CDR3 size distribution and reduced proliferative response toward mitogens. Most importantly, we unambiguously demonstrate that T cell abnormalities are not due to the absence of gp91phox protein expression and function into the NADPH-oxidase complex. Indeed, in T cells, ROS production is not mediated by gp91phox in physiological and in vitro conditions. Our results add to the knowledge of T-cell defects in CGD and highlight the necessity to extend the study of different cellular immune components in CGD patients in order to understand the complex pathogenic mechanism underlying the relations between innate and adaptive immunity.

\section{Acknowledgements}

The authors thank Maria Luisa Romiti for helping with TCRV-beta and lymphocyte proliferation assay: the Ospedale Pediatrico Bambino Gesù staff for their contribution to this study, CGD patients and their family.

\section{Author contributions}

MC designed and performed experiments, analysed the data, performed statistical analyses and wrote the paper; FC and GDM performed laboratory analysis and contributed to the data collection and revision of the manuscript; BG produced the LVV; AC, NC, DAP, PR provided patient's samples and clinical information, contributed to data discussion and drafting of the paper; AA supervised the project and revised the paper; AF supervised the project, designed the research, wrote and revised the paper. All authors critically revised and approved the final version of the manuscript.

\section{Conflict of interest}

All authors declare no conflict of interest.

\section{Funding}

This work was supported by: the European Commission - Advanced Cell-based Therapies for the treatment of Primary Immuno-Deficiency (HEALTH-F5-2010-261387, CELL-PID), the Italian TELETHON foundation (grant number GGP15109), Italian Ministero della Salute (NET2011-02350069). 


\section{References}

[1] M. Chiriaco, I. Salfa, G. Di Matteo, P. Rossi, A. Finocchi, Chronic granulomatous disease: clinical, molecular, and therapeutic aspects, Pediatr. Allergy Immunol. 27 (2016) 242-253, https://doi.org/10.1111/pai.12527 (Review).

[2] R.A. Seger, Chronic granulomatous disease: recent advances in pathophysiology and treatment, Neth. J. Med. 68 (2010) 334-340 (Review)

[3] C. Xie, T. Cole, C. McLean, J.C. Su, Association between discoid lupus erythematosus and chronic granulomatous disease-report of two cases and review of the literature Pediatr. Dermatol. 33 (Mar 2016) e114-e120, https://doi.org/10.1111/pde.12826.

[4] C.M. Cale, L. Morton, D. Goldblatt, Cutaneous and other lupus-like symptoms in carriers of X-linked chronic granulomatous disease: incidence and autoimmune serology, Clin. Exp. Immunol. 148 (2007) 79-84 (Review).

[5] S.S. De Ravin, N. Naumann, E.W. Cowen, J. Friend, D. Hilligoss, M. Marquesen, J.E Balow, K.S. Barron, M.L. Turner, J.I. Gallin, H.L. Malech, Chronic granulomatous disease as a risk factor for autoimmune disease, J. Allergy Clin. Immunol. 122 (2008) 1097-1103, https://doi.org/10.1016/j.jaci.2008.07.050.

[6] E.H. Verbon, J.A. Post, J. Boonstra, The influence of reactive oxygen species on cell cycle progression in mammalian cells, Gene 511 (2012) 1-6, https://doi.org/10. 1016/j.gene.2012.08.038 (Review).

[7] J.M. Matés, Effects of antioxidant enzymes in the molecular control of reactive oxygen species toxicology, Toxicology 153 (2000) 83-104 (Review).

[8] N. Cotugno, A. Finocchi, A. Cagigi, G. Di Matteo, M. Chiriaco, S. Di Cesare, P. Rossi, A. Aiuti, P. Palma, I. Douagi, Defective B-cell proliferation and maintenance of longterm memory in patients with chronic granulomatous disease, J. Allergy Clin. Immunol. 135 (2015) (753-61.e2) https://doi.org/10.1016/j.jaci.2014.07.012.

[9] M.M. Kamiński, D. Röth, P.H. Krammer, K. Gülow, Mitochondria as oxidative signaling organelles in T-cell activation: physiological role and pathological implications, Arch. Immunol. Ther. Exp. 61 (2013) 367-384, https://doi.org/10.1007/s00005013-0235-0 (Review).

10] L Simeoni, C. Thurm, A. Kritikos, A. Linkermann, Redox homeostasis, T cells and kidney diseases: three faces in the dark, Clin. Kidney J. 9 (1) (Feb 2016) 1-10, https://doi.org/10.1093/ckj/sfv135.

[11] A.V. Belikov, B. Schraven, L. Simeoni, T cells and reactive oxygen species, J. Biomed Sci. 22 (2015) 85, https://doi.org/10.1186/s12929-015-0194-3.

[12] M. Heltzer, A.F. Jawad, J. Rae, J.T. Curnutte, K.E. Sullivan, Diminished T cell numbers in patients with chronic granulomatous disease, Clin. Immunol. 105 (2002) $273-278$.
[13] M. Chiriaco, G. Farinelli, V. Capo, E. Zonari, S. Scaramuzza, G. Di Matteo, L.S. Sergi, M. Migliavacca, R.J. Hernandez, F. Bombelli, E. Giorda, A. Kajaste-Rudnitski, D. Trono, M. Grez, P. Rossi, A. Finocchi, L. Naldini, B. Gentner, A. Aiuti, Dual-regulated lentiviral vector for gene therapy of X-linked chronic granulomatosis, Mol. Ther. 22 (2014) 1472-1483, https://doi.org/10.1038/mt.2014.87.

[14] P. Palma, M.L. Romiti, C. Cancrini, S. Pensieroso, C. Montesano, S. Bernardi, M. Amicosante, S. Di Cesare, G. Castelli-Gattinara, B. Wahren, P. Rossi, Delayed early antiretroviral treatment is associated with an HIV-specific long-term cellular response in HIV-1 vertically infected infants, Vaccine 26 (40) (Sep 19 2008) 5196-5201.

[15] M.L. Romiti, C. Cancrini, G. Castelli-Gattinara, S. Di Cesare, P. Ciaffi, S. Bernardi, M.R. De Gasperi, E. Halapi, P. Rossi, Kinetics of the T-cell receptor CD4 and CD8 V beta repertoire in HIV-1 vertically infected infants early treated with HAART, AIDS 9 (15) (2001) 2075-2084.

[16] S.H. Jackson, S. Devadas, J. Kwon, L.A. Pinto, M.S. Williams, T cells express a phagocyte-type NADPH oxidase that is activated after T cell receptor stimulation, Nat. Immunol. 5 (8) (Aug 2004) 818-827.

[17] B. Kalyanaraman, V. Darley-Usmar, K.J. Davies, P.A. Dennery, H.J. Forman, M.B. Grisham, G.E. Mann, K. Moore, L.J. Roberts, H. Ischiropoulos, Measuring reactive oxygen and nitrogen species with fluorescent probes: challenges and limitations, Free Radic. Biol. Med. 52 (1) (Jan 1 2012) 1-6.

[18] K. Bedard, K.H. Krause, The NOX family of ROS-generating NADPH oxidases: physiology and pathophysiology, Physiol. Rev. 87 (2007) 245-313.

[19] A.V. Belikov, B. Schraven, L. Simeoni, TCR-triggered extracellular superoxide production is not required for T-cell activation, Cell Commun. Signal. 12 (2014) 50, https://doi.org/10.1186/s12964-014-0050-1.

[20] S. Salmen, D. Corte, L. Goncalves, L. Barboza, H. Montes, A. Calderón, L. Berrueta, CD40/CD40L expression in leukocytes from chronic granulomatous disease patients, APMIS 115 (2007) 939-947.

[21] Jameson SC1., Maintaining the norm: T-cell homeostasis, Nat. Rev. Immunol. 2 (2002) 547-556.

[22] J. Cachat, C. Deffert, S. Hugues, K.H. Krause, Phagocyte NADPH oxidase and specific immunity, Clin. Sci. (Lond.) 128 (10) (May 1 2015) 635-648.

[23] T.K. Teague, D. Hildeman, R.M. Kedl, T. Mitchell, W. Rees, B.C. Schaefer, J. Bender, J. Kappler, P. Marrack, Activation changes the spectrum but not the diversity of genes expressed by T cells, Proc. Natl. Acad. Sci. U. S. A. 96 (22) (Oct 26 1999) 12691-12696. 